\title{
The International Registry of patients with sarcopenia: applying research in sarcopenia to clinical practice
}

\author{
Dolores Sanchez-Rodriguez ${ }^{1,2,3,4}$ [ $\cdot$ Olivier Bruyère ${ }^{5}$
}

Received: 9 August 2018 / Accepted: 5 September 2018 / Published online: 17 September 2018

(c) European Geriatric Medicine Society 2018

Sarcopenia has been recently recognized as a disease for the purposes of coding (ICD-10-CM M62.84) and reimbursement $[1,2]$. This acknowledgement has important implications for researchers, as this disease is related to malnutrition and frailty [3], functional decline, high rate of hospitalization, loss of quality of life [4] and increased mortality [5], but the definitive diagnostic [6] and therapeutic strategies [2] to counteract this reversible condition remain a challenge [7].

The European Geriatric Medicine Society (EuGMS) is a leader in activities around sarcopenia in Europe and promoted the European Working Group on Sarcopenia in Older People (EWGSOP), which has proposed the most widely used algorithm for sarcopenia case finding and recommends assessment of sarcopenia in all geriatric patients and every healthcare setting [7].

The EuGMS founded the Special Interest Group (SIG) on Sarcopenia in September 2015, and published the first special issue on sarcopenia in European Geriatric Medicine in 2016 [8]; since then, the number of formal members of this interest group has increased to 74, making it the most extensive SIG of the EuGMS.

Staying in the lead of cutting-edge translational activities around sarcopenia, the EuGMS and the SIG on Sarcopenia are now launching the International Registry of patients with sarcopenia, among other initiatives, in an effort to bridge the

Dolores Sanchez-Rodriguez

97662@ parcdesalutmar.cat

1 Geriatrics Department, Parc Salut Mar, Barcelona, Spain

2 Rehabilitation Research Group, Institut Hospital del Mar d'Investigacions Mèdiques (IMIM), Barcelona, Spain

3 School of Medicine, Universitat Autònoma de Barcelona, Barcelona, Spain

4 Universitat Pompeu Fabra, Barcelona, Spain

5 Department of Public Health, Epidemiology and Health Economics, University of Liège, Quartier Hôpital, Avenue Hippocrate 13, CHUB23, 4000 Liège, Belgium gaps between research and clinical settings; and in line with the report from the International Conference on Frailty and Sarcopenia Research Task Force [2] and the World Health Organization's strategy to enhance initiatives towards optimal aging [9].

To the best of our knowledge, this EuGMS initiative is the first registry of patients with sarcopenia. In Geriatric medicine, there are very few registries. For example, the EURECCA International Comparison is an European registry on treatment and survival of older patients with colon cancer, involving six European countries and 50,000 patients in its first decade [10]; in Italy, the "Syncope and Dementia registry" is a prospective, observational multicenter study assessing the transient loss of consciousness in patients with dementia in usual care [11].

Other fields of medicine have a higher number of registries, with a wide range of policies on research access. A systematic review in nephrology identified 144 renal registries, of which 22 provided public access for publications or reports and only 13 made anonymized patient data available to the external researchers, either by application and review of the proposed data usage or by user fees [12]. In surgery, 18 clinical registries that focus on the surgical quality have been described [13]. In rheumatology [14], the main topic of registries is rare diseases; a systematic review identified 48 clinical registries about 23 different diseases, most of them classified as rare but they also include more common ones, such as psoriasis. In dermatology, 11 registries on survival after skin cancer, with a focus on the clinical adverse outcomes, have been described [15].

The ultimate goal of the International Registry of patients with sarcopenia is to unify the efforts and observations of clinicians and researchers worldwide, [2] and provide ready access to the data for research purposes. However, the registry also has intermediate objectives:

1. To identify the population where sarcopenia is being assessed; Currently, some of the populations likely to 
present sarcopenia have not been well studied, such as patients hospitalized in acute [16, 17] and post-acute [8] healthcare settings or living in long-term care facilities [18].

2. To assess the baseline characteristics of the patients with sarcopenia.

3. To evaluate and compare the tools and the cut-off values used in clinical practice to assess sarcopenia. The action research philosophy (i.e. applying research to clinical practice) [19] leaves information gaps about the assessment methods and the cut-off points to be used [6].

4. To compare the data between countries and world regions [20].

5. To create a collaborative network of researchers and clinical centers interested in combating sarcopenia. For example, 111 studies have been published from the data contained in the 18 clinical registries focused on the surgical quality [13]. In Spain, researchers who include data in FRENA (Factores de Riesgo y ENfermedad Arterial; in English, Risk Factors and Arterial Disease) form a collaborative group that has co-authored 16 publications to date from the registry data $[21,22]$.

In the future, this registry could perhaps be used for quality assurance, as is the case in Germany for some cancer registries [23]. The centers involved are certified and included in the national comparative analysis of adherence to guidelines. They also actively contribute to many research projects, providing data on cancer etiology, screening programs, patient care and clinical outcomes of the population-based studies [23].

The design and development of this Registry incorporated evidence from the epidemiological studies on sarcopenia [24]. The data collection was modeled after existing registries in other disciplines, in an effort to encourage participation and optimize management of the contributed data. Currently, the data collected (as available for each participating center) include muscle mass, strength and physical performance as well as the demographic and socio-economic information.

This is an International Registry because the variables requested meet the criteria of most existing definitions: European Working Group on Sarcopenia in Older People [7] and the 2018 consensus, in process of publication, Asian Working Group for Sarcopenia (AWGS) [25], International Working Group on Sarcopenia (IWGS) [26], Foundation for the National Institutes of Health definition (FNIH) [27], Society on Sarcopenia, Cachexia and Wasting Disorders (SSCWD) [28], European Society for Clinical Nutrition and the Metabolism-Special Interest Group [29]. Moreover, all races/ethnicities and countries have been represented and users decide and specify the cut-off points used. The term "European", used twice in the website, should not limit the use of the Registry worldwide.

The initial reception by researchers from countries outside of Europe is promising and the Publication plan for projects proposed by the users is a work in progress. Collaborative partnerships with researchers and clinicians from scientific societies worldwide are expected and welcome.

One of the premises for developing the International Registry of patients with sarcopenia was that the value of the Registry must compensate for the effort and time invested in data recording. All participants in the Registry will contribute to the knowledge on sarcopenia. In addition, those who substantially contribute to the Registry could submit a research proposal based on the data of the registry that will be checked and approved by a scientific committee. These active members of the Registry will also be eligible to join the Registry's network of active researchers, including leaders of the SIG on Sarcopenia, EuGMS and other key groups working to improve the geriatric care.

Participation requires completion of a user profile (https:// www.e-clinical.org/sarcopenia/), which includes information on the clinical setting, assessment methods and the cut-off points used. After this brief ( 5-min) step, patient data can be entered freely, with no limitations on the number of cases included.

Measures to ensure the quality of data recording as much as possible have been adopted. Internal caution measures related to the structure of the website were planned during the design and development of the Registry. External caution measures involve the Working Group on the International Registry and the eventual network of users. These measures are detailed as follows:

1. Internal caution measures: the design and development of the Registry website.

1.1 Each researcher is identified individually and is responsible for his or her own data entry and procedures; when a new user from a center already listed in the registry accesses the platform, this individual must again enter the identifying details for that center.

1.2 There is a contract in the first page of the Registry; by saving this form, the investigator agrees to the terms and conditions. These include, among others, being responsible for "the objectives, basic methodology and results"; moreover, after a data entry session, the researcher reaffirms this contract by responding to the following prompt: "I hereby confirm that the data contained in this visit are correct and complete".

1.3 To ensure the veracity of data, the design of the Registry makes it mandatory to enter the complete 
patient data "one by one", avoiding approaches such as uploading the data fields completed offline (e.g., in Excel or.css format) for multiple patients.

2. External caution measures: the Working Group on the International Registry

2.1 The members of the Working Group of the International Registry have access to the data and the affiliation of the users and check them periodically; those users with outlier or discordant data will be contacted.

2.2 Only centers and researchers that have included patient data will have access to the Registry data set; proposals for the use of data will be assessed by the Working group and included in the Publication Plan of the Registry, if approved.

\section{Double-control caution measure}

3.1 Only registered users can enter or modify their own data. The members of the Working Group with access to the data and the affiliations cannot modify other users' data. If a change is required, the Working Group must contact the user directly, or the user can contact the support team.

The International Registry of patients with sarcopenia is expected to become a long-term source of data to inform the best-practice care of our aging population and to develop international research projects to advance our knowledge of this debilitating but reversible disease.

\begin{abstract}
Acknowledgements The authors acknowledge Francesco Landi and Jürgen Bauer (Presidents, SIG Sarcopenia), and Stephania Maggi and Finbarr Martin (Immediate Past President and President of the EuGMS), as key personnel in developing the Registry. Dolores Sanchez-Rodriguez and Olivier Bruyère, as current co-leaders of the Registry initiative, also acknowledge Alfonso Cruz-Jentoft for his work as founding chair of SIG on Sarcopenia and prime mover of the Registry, and Charlotte Beaudart, Antonio Cherubini, Médéa Locquet, and Andrea Hovenier for their valuable contribution as European Working Group on the International Registry of patients with sarcopenia. The authors wish to acknowledge Abbott, Nutricia, and Nestlé for grants to cover the expenses of the technical support needed to launch the Registry. The authors appreciate the unfailing editorial assistance provided by Elaine M. Lilly, Ph.D.
\end{abstract}

Author contributions DSR and OB wrote the manuscript and did literature review and OB corrected the manuscript. All co-authors read and approved the final version of the manuscript.

\section{Compliance with ethical standards}

Conflict of interest All authors declare they do not have any financial and personal relationships with other people or organizations that could inappropriately influence their work. Researchers and the con- tent of the Registry were not conditioned or influenced in any manner by Abbott, Nutricia, and Nestlé, which funded the project. Data entered are treated in accordance with the provisions of the applicable data protection law in Spain and the General Data Protection Regulation (GDPR) (EU) 2016/679 of the European Parliament and Council, dated the 27 April of 2016.

Ethical approval This study does not contain any studies with human participants performed by any of the authors.

Informed consent For this type of study formal consent is not required.

\section{References}

1. Anker SD, Morley JE, von Haehling S (2016) Welcome to the ICD-10 code for sarcopenia. J Cachexia Sarcopenia Muscle 7(5):512-514

2. Vellas B, Fielding RA, Bens C, Bernabei R, Cawthon PM, Cederholm $\mathrm{T}$ et al (2018) Implications of ICD-10 for Sarcopenia Clinical Practice and Clinical Trials: report by the International Conference on Frailty and Sarcopenia Research Task Force. J Frailty Aging 7(1):2-9

3. Cederholm T, Bosaeus I, Barazzoni R, Bauer J, Van Gossum A, Klek S et al (2015) Diagnostic criteria for malnutrition-an ESPEN consensus statement. Clin Nutr 34(3):335-340

4. Beaudart C, Reginster JY, Petermans J, Gillain S, Quabron A, Locquet $\mathrm{M}$ et al (2015) Quality of life and physical components linked to sarcopenia: the SarcoPhAge study. Exp Gerontol 69:103-110

5. Beaudart C, Zaaria M, Pasleau F, Reginster J-Y, Bruyère O (2017) Health outcomes of sarcopenia: a systematic review and metaanalysis. PLoS One. 12(1):e0169548 (Wright JM, editor)

6. Rodríguez-García WD, García-Castañeda L, Vaquero-Barbosa N, Mendoza-Núñez VM, Orea-Tejeda A, Perkisas S et al (2018) Prevalence of dynapenia and presarcopenia related to aging in adult community-dwelling Mexicans using two different cut-off points. Eur Geriatr Med 9(2):219-225

7. Cruz-Jentoft AJ, Baeyens JP, Bauer JM, Boirie Y, Cederholm T, Landi F et al (2010) Sarcopenia: European consensus on definition and diagnosis. Age Ageing 39(April):412-423

8. Sánchez-Rodríguez D, Calle A, Contra A, Ronquillo N, Rodríguez-Marcos A, Vázquez-Ibar O et al (2016) Sarcopenia in postacute care and rehabilitation of older adults: a review. Eur Geriatr Med. 7(3):224-231

9. World Health Organisation (2016) Global strategy and action plan on ageing and health (2016-2020). World Health Organisation, Geneva

10. Vermeer NCA, Claassen YHM, Derks MGM, Iversen LH, van Eycken E, Guren MG et al (2018) Treatment and survival of patients with colon cancer aged 80 years and older: a EURECCA international comparison. Oncologist. https://doi.org/10.1634/ theoncologist.2017-0551

11. Ungar A, Mussi C, Nicosia F, Ceccofiglio A, Bellelli G, Bo M et al (2015) The "syncope and dementia" study: a prospective, observational, multicenter study of elderly patients with dementia and episodes of "suspected" transient loss of consciousness. Aging Clin Exp Res 27(6):877-882

12. Liu FX, Rutherford P, Smoyer-Tomic K, Prichard S, Laplante S (2015) A global overview of renal registries: a systematic review. BMC Nephrol. 16(1):31

13. Stey AM, Russell MM, Ko CY, Sacks GD, Dawes AJ, Gibbons MM (2015) Clinical registries and quality measurement in surgery: a systematic review. Surg (United States). 157(2):381-395 
14. Beukelman T, Kimura Y, Ilowite NT, Mieszkalski K, Natter MD, Burrell G et al (2017) The new Childhood Arthritis and Rheumatology Research Alliance (CARRA) registry: design, rationale, and characteristics of patients enrolled in the first 12 months. Pediatr Rheumatol Online J 15(1):30

15. DiMarco G, Hill D, Feldman SR (2016) Review of patient registries in dermatology. J Am Acad Dermatol. 75:824-829

16. Bianchi L, Abete P, Bellelli G, Bo M, Cherubini A, Corica F et al (2017) Prevalence and Clinical Correlates of Sarcopenia, Identified According to the EWGSOP Definition and Diagnostic Algorithm, in Hospitalized Older People: the GLISTEN Study. J Gerontol A Biol Sci Med Sci 72(11):1575-1581

17. Martone AM, Bianchi L, Abete P, Bellelli G, Bo M, Cherubini A et al (2017) The incidence of sarcopenia among hospitalized older patients: results from the Glisten study. J Cachexia Sarcopenia Muscle 8(6):907-914

18. Mesquita AF, da Silva EC, Eickemberg M, Roriz AKC, BarretoMedeiros JM, Ramos LB (2017) Factors associated with sarcopenia in institutionalized elderly. Nutr Hosp 34(2):345-351

19. Beauchet O, Fantino B, Annweiler C (2012) The 'action-research' philosophy: from bedside to bench, to bedside again. Int J Clin Pract 66(5):517

20. Ethgen $\mathrm{O}$, Beaudart C, Buckinx F, Bruyère $\mathrm{O}$, Reginster JY (2017) The future prevalence of sarcopenia in Europe: a claim for public health action. Calcif Tissue Int 100(3):229-234

21. Monreal M, Alvarez L, Vilaseca B, Coll R, Suárez C, Toril J et al (2008) Clinical outcome in patients with peripheral artery disease. Results from a prospective registry (FRENA). Eur J Intern Med 19(3):192-197

22. Altes P, Perez P, Esteban C, Sánchez Muñoz-Torrero JF, Aguilar E, García-Díaz AM et al (2017) Raised fibrinogen levels and outcome in outpatients with peripheral artery disease. Angiology 7:000331971773972

23. Holleczek B, Katalinic A (2017) Toward a comprehensive cancer registration in Germany. Eur J Cancer Prev 26:S132-S138

24. Locquet M, Beaudart C, Reginster J-Y, Petermans J, Gillain S, Quabron A et al (2017) Prevalence of concomitant bone and muscle wasting in elderly women from the SarcoPhAge cohort: preliminary results. J Frailty Aging 6(1):18-23

25. Chen LK, Liu LK, Woo J et al (2014) Sarcopenia in Asia: consensus report of the Asian Working Group for Sarcopenia. J Am Med Dir Assoc 15:95-101

26. Fielding RA, Vellas B, Evans WJ et al (2011) Sarcopenia: an undiagnosed condition in older adults. Current consensus definition: prevalence, etiology, and consequences. International working group on sarcopenia. J Am Med Dir Assoc 2:249-256

27. McLean RR, Shardell MD, Alley DE et al (2014) Criteria for clinically relevant weakness and low lean mass and their longitudinal association with incident mobility impairment and mortality: the foundation for the National Institutes of Health (FNIH) sarcopenia project. J Gerontol A Biol Sci Med Sci 69:576-583

28. Morley JE, Abbatecola AM, Argiles JM et al (2011) Society on Sarcopenia, Cachexia and Wasting Disorders Trialist Workshop. Sarcopenia with limited mobility: an international consensus. J Am Med Dir Assoc 12:403-409

29. Muscaritoli M, Anker SD, Argilés J et al (2010) Consensus definition of sarcopenia, cachexia and pre-cachexia: joint document elaborated by Special Interest groups (SIG) "cachexia-anorexia in chronic wasting diseases" and "nutrition in geriatrics". Clin Nutr 29:154-159 